\title{
Estudio experimental sobre el secado de bagazo de caña de azúcar panelera
}

\author{
Experimental study of the drying of sugar cane bagasse
}

\author{
J. A. Villalba-Vidales iD, N. Arzola-de-la-Peña iD
}

\begin{abstract}
Optimizing biomass drying to improve combustion processes is one of the main tools available to small and medium agroindustrial producers in rural areas of Colombia to be competitive. For this reason, the development of experimental studies becomes a priority to improve the competitiveness of the rural sector in Colombia. In this way this paper shows the development of the experimental study of drying kinetic of panelera's sugar cane bagasse, as well as its contrast with mathematical models used for the prediction of the drying kinetics. The results obtained can be useful as a reference for the effective design of sugarcane bagasse drying systems.
\end{abstract}

Index Terms - biomass, drying kinetic, experimental model of drying, panela's sector, sugarcane bagasse.

Resumen- Optimizar el secado de biomasa para mejorar los procesos de combustión, se constituye en una de las principales herramientas con las que cuentan los pequeños y medianos productores agroindustriales en las áreas rurales de Colombia para ser competitivos. Por esta razón el desarrollo de estudios experimentales se convierte en una prioridad para mejorar la competitividad del sector rural en Colombia. En este sentido el presente documento muestra el desarrollo del estudio de la cinética experimental del bagazo de caña panelera, así como su contraste con modelos matemáticos usados para le predicción de la cinética de secado. Los resultados obtenidos pueden ser útiles como referencia para el diseño efectivo de sistemas de secado de bagazo de caña de azúcar.

Palabras claves - bagazo de caña, biomasa, cinética de secado, experimental de secado, modelo sector panelero.

\section{INTRODUCTION}

$\mathrm{E}$ 1 proceso de secado es usado por el hombre desde que fue consciente que los alimentos podrían ser preservados mediante la reducción del contenido de humedad [1]. Más recientemente el proceso de secado ha cobrado otra relevancia debido a que se constituye en un pre-proceso necesario en el mejoramiento de la biomasa para uso como combustible [2]. Una de las principales desventajas cuando se requiere secar biomasa, es su baja temperatura de autoignición, que se

Este manuscrito fue enviado el 26 de abril de 2018 y aceptado el 15 de marzo de 2019.

J. A. Villalba-Vidales Profesor en la Fundación Universitaria los Libertadores, Carrera 16 No. 63 A - 68, Bogotá, Colombia (e-mail javillalbav01@libertadores.edu.co). encuentra entre $260^{\circ} \mathrm{C}$ y $280^{\circ} \mathrm{C}$. Lo anterior limita la temperatura máxima del medio de secado, en especial cuando se trabajan con aire. Debe considerarse además que las temperaturas elevadas generan mayores emisiones de material orgánico volátil [3], [4], [5].

Existen diversos estudios de secado de biomasa [6], destacándose el de Bartzanas et al. [7] los cuales desarrollaron un estudio de la cadena de pasto, incluyendo el estudio experimental y modelado matemático del proceso de secado en campo. Esto con el fin de mejorar los procesos asociados de almacenamiento y transporte de la biomasa. Por su parte, Ivanonva et al. [8] desarrollaron un estudio experimental de secado de biomasa. Para ello usaron una unidad experimental que consta de una cámara de secado, que posee una cámara de mezclado de aire y un colector solar. El secador puede ser operado mediante intercambiadores de calor con la caldera o mediante el sistema de calefacción solar.

Rada et al. [5] estudiaron el bio-secado de diferentes materiales dentro de los que se encuentran residuos de la industria vinícola, aguas residuales y desechos municipales mezcladas en diferentes proporciones. Para ello desarrollaron un secador experimental, el cual consistía en un reactor adiabático soportado sobre una balanza electrónica con el fin de monitorear el secado de la biomasa.

Mazutti et al. [9] llevaron a cabo pruebas experimentales de secado de bagazo de caña, recurriendo a un secador a escala de laboratorio. El secador consiste básicamente de tres unidades. Un ventilador que provee el aire deseado; un sistema de calentamiento con una potencia de $3 \mathrm{~kW}$ y un sistema de control. El material por secar es esparcido en capa delgada en una bandeja de aluminio. El experimento se llevó a cabo bajo cuatro condiciones de temperatura. $30^{\circ} \mathrm{C}, 35^{\circ} \mathrm{C}, 40^{\circ} \mathrm{C}$ y $45^{\circ} \mathrm{C}$, empleando flujos volumétricos de 2,0 y $3,0 \mathrm{~m}^{3} \mathrm{~h}^{-1}$. El espesor de la capa fue de aproximadamente $10 \mathrm{~mm}$. Para medir el peso del material durante el secado, la muestra fue retirada de la cámara de secado y pesada en una balanza. Por otra parte, Sosa-Arnao et al. [10] realizaron una revisión completa de estudios experimentales de secado de bagazo de caña. En dicha revisión se presentan diversas tecnologías de secado, haciendo énfasis

N. Arzola de la Peña Profesor Titular en la Universidad Nacional de Colombia, Departamento de Ingeniería Mecánica y Mecatrónica, Cra. 30 No. 45-03, Bogotá, Colombia (e-mail narzola@unal.edu.co). 
en los resultados de humedad final promedio en las muestras. Destacándose el hecho que el primer estudio de secado de bagazo fue desarrollado por Kerr en 1910. Para esta investigación el secador fue construido en acero de 1,2 m x 1,8 $\mathrm{m}$ de sección transversal y $6 \mathrm{~m}$ de altura. Trabajaba a contracorriente y permitía reducir el contenido de humedad del bagazo de caña de $54,3 \%$ a $46,4 \%$ en b.h. [10].

En el presente trabajo se determina experimentalmente la cinética de secado de bagazo de caña panelera proveniente de un trapiche típico del municipio San José de Isnos del departamento de Huila. Para ello se construyó una unidad de secado novedosa a escala de laboratorio, con el fin de estudiar el comportamiento del material a secar y del medio de secado a lo largo del proceso. Los resultados obtenidos fueron procesados estadísticamente con el fin de evaluar las diferencias presentes para cada tratamiento. Por último, los resultados experimentales se compararon con resultados obtenidos mediante la solución de modelos matemáticos obtenidos por otros investigadores.

\section{MATERIALES Y Metodos}

\section{A. Unidad experimental}

El estudio de secado de bagazo de caña panelera se llevó a cabo en un secador a escala de laboratorio novedoso desarrollado específicamente para la presente investigación. La disposición de los componentes que conforman la unidad experimental se muestra en la Fig. 1, la misma está dividida en tres secciones. Primero se tiene una unidad de calentamiento, la cual consta de un ventilador axial con un diámetro de 9" con un flujo promedio variable entre 470 y $490 \mathrm{~m}^{3} \mathrm{~h}^{-1}$ (flujo de aire en vacío) adicional a esto, cuenta con un banco de resistencias con una potencia de $9 \mathrm{~kW}$. Luego se encuentra la cámara de secado. Dicha cámara está provista de dos mallas metálicas, las cuales permiten la entrada y salida del aire de secado en la dirección axial. La cámara de secado además cuenta con cinco sondas con sensores instalados (de humedad y temperatura), que permiten conocer las condiciones del medio de secado. Por último, existe un módulo de adquisición de datos, el cual se encarga de recolectar la información de los sensores ubicados a lo largo de la unidad experimental.

Los sensores son del tipo DTH11 y se encuentran distribuidos de la siguiente forma: Sensor S1 a la salida del sistema de calentamiento (con el fin de medir las propiedades a la entrada de la cámara de secado). Sensores S2, S3 y S4: distribuidos uniformemente a lo largo de la cámara de secado (con el fin de determinar el perfil de temperatura y humedad dentro del colchón de biomasa). Estos sensores tienen la posibilidad de ser colocados en diferentes posiciones dentro de la cámara de secado. Sensor S5 a la salida de la cámara de secado (con el fin de medir las propiedades del aire justo a la salida de la cámara de secado). Estos sensores tienen un rango de funcionamiento para temperatura entre $0-50^{\circ} \mathrm{C}$ y un rango de funcionamiento para humedad entre $20-90 \%$ HR.

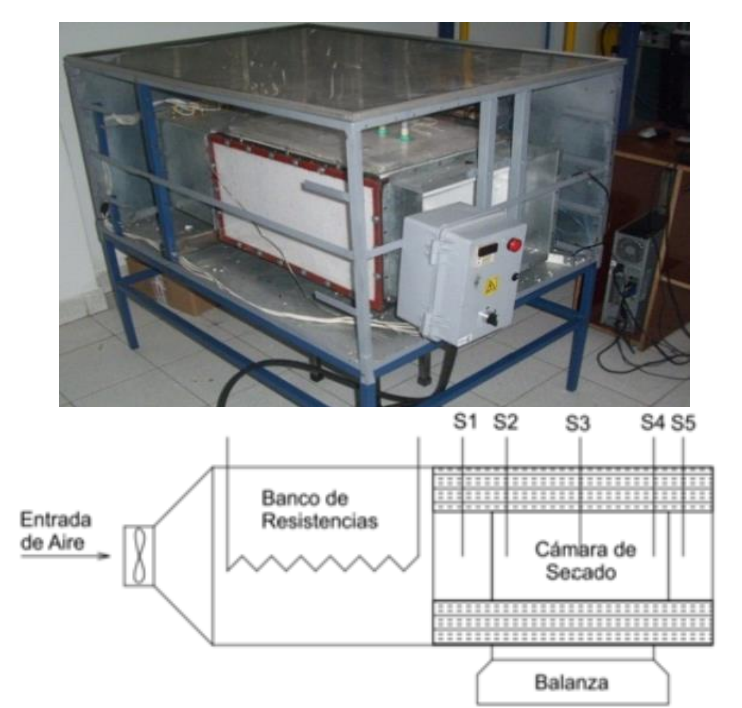

Fig. 1. Unidad experimental de secado y su diagrama esquemático interno.

La unidad experimental posee una balanza electrónica TAC sobre la cuál descansa la cámara de secado. La balanza esta provista de un puerto serial RS232 el cuál es conectado al computador permitiendo el registro de la variación del peso. La balanza cuenta con un rango de medida de entre 0 a $30 \mathrm{~kg}$ y una resolución de $\pm 1 \mathrm{~g}$.

La comunicación de los sensores para la medición de temperatura y humedad DTH11 se lleva a cabo mediante un microcontrolador Arduino Mega ATmega1280, el cual se acopla mediante puerto USB con el computador a través de una aplicación desarrollada en Labview. Esta aplicación permite la adquisición de los datos de peso de la biomasa, temperatura y humedad para las cinco posiciones, y su posterior registro como un archivo .xls al finalizar la corrida experimental. De esta forma, las corridas experimentales arrojan información sobre los perfiles de temperatura y humedad dentro de la cámara de secado durante el tiempo de duración de los ensayos; así como de la disminución del peso de la muestra producto a la disminución del contenido de humedad producto al proceso de secado.

\section{B. Preparación de las muestras de bagazo de caña de azúcar}

El bagazo de caña panelera se obtuvo en el municipio de San José de Isnos del departamento de Huila, de una de las casas de secado de un trapiche típico del departamento del Huila. Una vez recibido el material fue dejado en reposo en un lugar fresco, con el fin de permitir que la humedad se homogeneizara en todo el material. El tamaño del material se mantuvo igual a como sale del trapiche.

\section{1) Triturado de la muestra}

Antes de someter la muestra al proceso de secado fue necesario reducir la longitud de la fibra. Para ello se usó una picadora y un tamiz para obtener tres tamaños diferentes de la fibra. Una vez obtenidos los diferentes tamaños de fibra se usó una aplicación del Software Matlab denominada measuretool_1.13.m. La cual permite de manera gráfica 
determinar la longitud media de la fibra. Para cada una de las muestras se tomaron aproximadamente 40 mediciones, las cuales fueron procesadas estadísticamente obteniendo los resultados que se presentan en la Tabla 1 .

TABLA I

Resumen de La Medición De la Longitud De Fibra Del Bagazo De CAÑa De AZÚCAR (MEDidAs EN MM)

\begin{tabular}{llll}
\hline \hline Muestra & $\begin{array}{l}\text { Longitud } \\
\text { promedio }\end{array}$ & $\begin{array}{l}\text { Desviación } \\
\text { estándar }\end{array}$ & $\begin{array}{l}\text { Rango de } \\
\text { medidas }\end{array}$ \\
1 & 5,74 & 0,64 & $3,80-7,67$ \\
2 & 24,39 & 6,45 & $5,014-43,77$ \\
3 & 53,77 & 10,86 & $21,17-86,36$ \\
\hline \hline
\end{tabular}

De la misma forma se llevó a cabo el proceso de medición de la sección transversal de la fibra, para lo cual se llevó a cabo el mismo procedimiento que en la medición de la longitud de la fibra. Los resultados se presentan en la Tabla 2. Para cada uno de los casos se aplicó una prueba de normalidad con el fín de verificar la veracidad de los resultados. También fue demostrada la relación proporcional entre la longitud y la sección transversal de las fibras del material.

TABLA II

Resumen De la Medición Del Diámetro De Fibra Del Bagazo De CAÑA DE AZÚCAR (MEDIDAS EN MM)

\begin{tabular}{llll}
\hline \hline Muestra & Longitud & $\begin{array}{l}\text { Desviación } \\
\text { Estándar }\end{array}$ & Rango de Medidas \\
& Promedio & 0,308 & $0,049-1,899$ \\
1 & 0,97 & 0,270 & $0,804-2,426$ \\
2 & 1,62 & 0,540 & $1,485-4,725$ \\
3 & 3,11 & \\
\hline \hline
\end{tabular}

\section{2) Determinación de la humedad inicial de las muestras}

Después de homogeneizar el material se determinó la humedad de la muestra. Para ello se seleccionaron tres muestras al azar del total de la muestra. Con el fin de promediar la humedad en la muestra. Las muestras seleccionadas se depositan sobre contenedores con el fin de ser llevados a una mufla, donde es calentada a $100^{\circ} \mathrm{C}$. La muestra se retira periódicamente, hasta que el peso no presente variaciones significativas. La humedad inicial promedio de las muestras fue de $12,34 \%$ b.h.

Una vez obtenidos los resultados de humedad, se determinó la cantidad de agua a adicionar para lograr los tres niveles de contenido de humedad necesarios. Para cada caso la adición de agua se llevó a cabo entre 18 y 24 horas antes del ensayo de secado. La humidificación se llevó a cabo con un aerosol y la muestra fue sellada con el fin de evitar pérdidas de humedad posteriores.

\section{3) Temperatura del medio de secado}

La temperatura del medio de secado para la presente investigación se seleccionó teniendo en cuenta el rango de temperatura más común utilizado por otros investigadores. Este rango es bastante amplio y está comprendido entre los $20^{\circ} \mathrm{C}$ para sistemas pasivos y $\operatorname{los} 65^{\circ} \mathrm{C}$ para sistemas de convección forzada [11]. Adicional a esto, Mazutti et al. [9] plantea en su estudio sobre el secado de bagazo de caña un rango de temperatura entre $35^{\circ} \mathrm{C}$ y $60^{\circ} \mathrm{C}$. Por otro lado, también se deberá considerar las limitaciones técnicas propias del equipo secador implementado. Finalmente, con base a este grupo de criterios se selecciona un rango de temperatura entre $35^{\circ} \mathrm{C}$ y $60^{\circ} \mathrm{C}$.

\section{Arreglo experimental}

El número de corridas experimentales se determinó mediante la aplicación de un diseño experimental factorial completo, el cual consta de dos factores $y$ tres niveles. Los factores experimentales considerados fueron la temperatura del medio de secado (aire) y la longitud de la fibra de bagazo de caña de azúcar. Adicional a esto se decidió desarrollar tres repeticiones por cada experimento (replicas). De tal forma que el número total de corridas experimentales fue de 27. La duración del experimento se fijó a partir del desarrollo experimental de Mazutti et al. [9], el cual desarrolló un proceso de secado de bagazo de caña con tratamientos superficiales, estableciendo un tiempo de duración de las pruebas de secado de 180 min. Por otro lado, las respuestas a medir fueron los perfiles de temperatura en la dirección axial del colchón de bagazo y el porcentaje de secado logrado en las muestras experimentales.

\section{RESULTADOS Y DiSCUSIÓN}

El estudio de los resultados se lleva a cabo en dos fases. En la primera se estudiará la influencia de cada una de las variables independientes (tamaño de fibra y temperatura del medio de secado) en el contenido de humedad final, la cual se puede ver en los resultados presentes en la Tabla 3 y en las Fig. 2 y 3 , respectivamente. Por último, se lleva a cabo un análisis de la cinética de secado, analizando el comportamiento de la humedad global de la muestra a lo largo del ensayo experimental. Esto se puede visualizar claramente en las Fig. 4 a 6.

Como se puede observar en la tabla 3 de humedad final, existe una mayor influencia del tamaño de fibra en los resultados obtenidos. También, se aprecia que para el nivel inferior de tamaño de fibra $(\mathrm{L}=6 \mathrm{~mm})$ no existen variaciones significativas de la humedad con la temperatura.

TABLA III

Resultados Experimentales Del CONTENIdo De Humedad FinAl DEL

\begin{tabular}{|c|c|c|c|c|c|}
\hline \multirow{3}{*}{$\begin{array}{l}\text { Tempe } \\
\text { ratura } \\
{\left[{ }^{\circ} \mathrm{C}\right]}\end{array}$} & Longitud & Corrida & Corrida & Corrida & Promedio \\
\hline & de Fibra & 1 & 2 & 3 & \\
\hline & {$[\mathrm{mm}]$} & & & & \\
\hline \multirow{3}{*}{35} & 6 & 45,23 & 45,32 & 45,32 & 45,29 \\
\hline & 24 & 24,84 & 29,38 & 19,61 & 24,61 \\
\hline & 54 & 11,42 & 3,02 & 5,78 & 6,74 \\
\hline \multirow{3}{*}{45} & 6 & 42,72 & 43,33 & 44,46 & 43,50 \\
\hline & 24 & 1,13 & 20,98 & 14,17 & 17,09 \\
\hline & 54 & 5,94 & 6,49 & 0,53 & 4,32 \\
\hline \multirow{3}{*}{60} & 6 & 41,50 & 42,39 & 43,60 & 42,50 \\
\hline & 24 & 2,74 & 2,20 & 15,47 & 6,80 \\
\hline & 54 & 1,55 & 1,81 & 1,65 & 1,67 \\
\hline
\end{tabular}




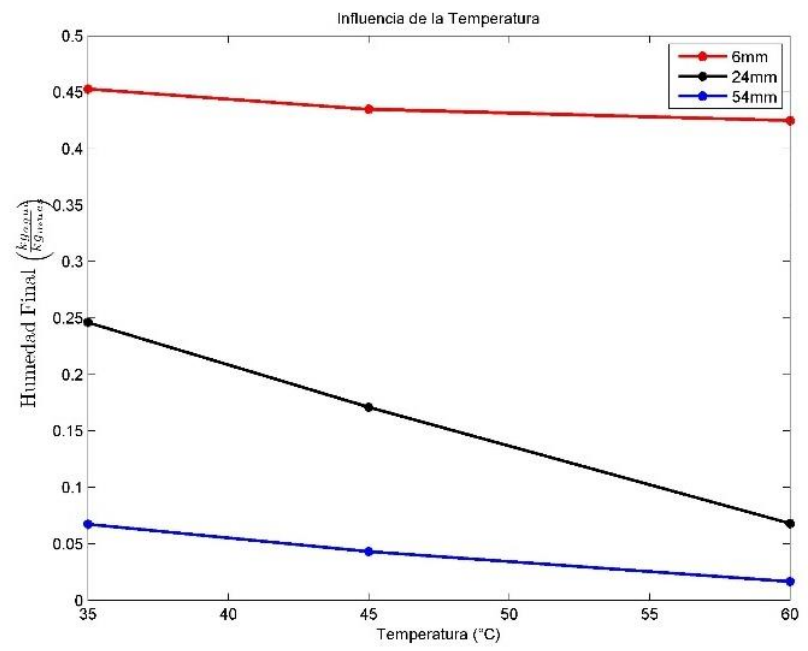

Fig. 2. Influencia de la temperatura en la humedad final de la muestra.

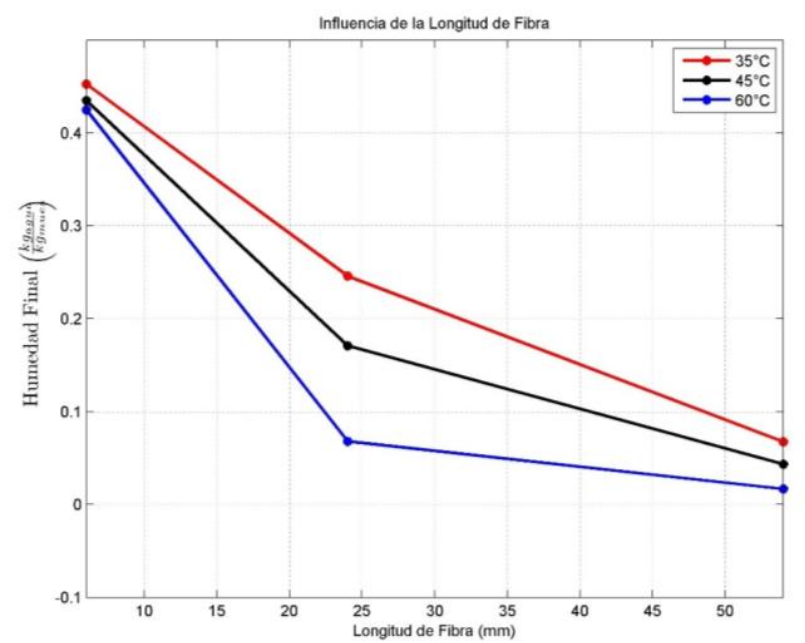

Fig. 3. Influencia del tamaño de fibra en la humedad final de la muestra.

A los resultados obtenidos se les aplicaron pruebas de hipótesis con el fin de verificar si existe diferencias estadísticamente significativas entre los resultados de las humedades finales de cada experimento. Inicialmente se desarrolló un análisis de varianza (ANOVA), partiendo de la hipótesis nula que no existen diferencias significativas en las medias de cada tratamiento. Para cada caso un valor cercano a cero significa la existencia de diferencias en las medias de la humedad final en al menos uno de los tratamientos. En la Tabla 4 se evidencia que los valores del estadígrafo $\mathrm{p} \approx 0$ muestran que existen diferencias significativas en las medias al menos para un tratamiento tanto en las filas (temperatura del medio de secado), como en las columnas (tamaño de la fibra de biomasa).

TABLA IV

RESUltados ANÁLISIS ANOVA

\begin{tabular}{llllll}
\hline Fuente & SS & Df & MS & F & Prob>F \\
Columnas & 0,73961 & 2 & 0,36981 & 269,08 & 0 \\
Filas & 0,03302 & 2 & 0,01651 & 12,02 & 0,0005 \\
Interacción & 0,01998 & 4 & 0,00499 & 3,63 & 0,0244 \\
Error & 0,02474 & 18 & 0,00137 & & \\
Total & 0,81735 & 26 & & & \\
\hline \hline
\end{tabular}

La otra prueba de hipótesis desarrollada es la t de Student, la cual prueba la existencia de diferencias estadísticamente significativas entre los resultados de contenido de humedad final para cada uno de los tratamientos, pero esta vez por separado. Este análisis parte de la hipótesis nula que no existen diferencias significativas entre las muestras con distinto tratamiento. En la Tabla 5 se presenta la codificación de los experimentos a ser comparados.

TABLA V

\begin{tabular}{ll} 
CODIFICACIÓN DE MUESTRAS PARA PRUEBA DE STUDENT \\
\hline \hline Niveles de los factores & Codificación \\
$\mathrm{T}=35^{\circ} \mathrm{C}$ y $\mathrm{L}=6 \mathrm{~mm}$ & 1 \\
$\mathrm{~T}=35^{\circ} \mathrm{C}$ y $\mathrm{L}=24 \mathrm{~mm}$ & 2 \\
$\mathrm{~T}=35^{\circ} \mathrm{C}$ y L $=54 \mathrm{~mm}$ & 3 \\
$\mathrm{~T}=45^{\circ} \mathrm{C}$ y $\mathrm{L}=6 \mathrm{~mm}$ & 4 \\
$\mathrm{~T}=45^{\circ} \mathrm{C}$ y $\mathrm{L}=24 \mathrm{~mm}$ & 5 \\
$\mathrm{~T}=45^{\circ} \mathrm{C}$ y $\mathrm{L}=54 \mathrm{~mm}$ & 6 \\
$\mathrm{~T}=60^{\circ} \mathrm{C}$ y $\mathrm{L}=6 \mathrm{~mm}$ & 7 \\
$\mathrm{~T}=60^{\circ} \mathrm{C}$ y $\mathrm{L}=24 \mathrm{~mm}$ & 8 \\
$\mathrm{~T}=60^{\circ} \mathrm{C}$ y $\mathrm{L}=54 \mathrm{~mm}$ & 9 \\
\hline \hline
\end{tabular}

En la Tabla 6 se presentan los resultados de la influencia de la longitud de la fibra de la biomasa en la humedad final; mientras que en la Tabla 7 se presentan los resultados de la influencia de la temperatura del medio de secado.

TABLA VI

VALORES DE LA PRUEBA T DE STUDENT PARA LONGITUD DE FIBRA DE LA

\begin{tabular}{|c|c|c|c|c|c|}
\hline \multicolumn{6}{|c|}{ BIOMASA } \\
\hline Bloques & $\bar{h}$ & Valor $\mathrm{p}$ & $\overline{\mathrm{GdL}}$ & Estadístico & $\begin{array}{l}\text { Desviación } \\
\text { Estándar }\end{array}$ \\
\hline 1 vs 4 & 1 & 0,0251 & 4 & 3,3891 & 0,0063 \\
\hline 1 vs 7 & 1 & 0,0101 & 4 & 4,5918 & 0,0075 \\
\hline 4 vs 7 & 0 & 0,2744 & 4 & 1,2655 & 0,0097 \\
\hline 2 vs 5 & 0 & 0,0964 & 4 & 2,1648 & 0,0425 \\
\hline 2 vs 8 & 1 & 0,0263 & 4 & 3,4412 & 0,0634 \\
\hline 5 vs 8 & 0 & 0,0979 & 4 & 2,1507 & 0,0568 \\
\hline 3 vs 6 & 0 & 0,4806 & 4 & 0,7768 & 0,0382 \\
\hline 3 vs 9 & 0 & 0,1098 & 4 & 2,0492 & 0,0303 \\
\hline 6 vs 9 & 0 & 0,2371 & 4 & 1,3894 & 0,0233 \\
\hline
\end{tabular}

TABLA VII

VALORES DE LA PRUEBA T DE STUDENT PARA TEMPERATURA DEL FLUJO DE

\begin{tabular}{lccccc}
\multicolumn{5}{c}{ ENTRADA } \\
\hline \hline Bloques & $\mathrm{h}$ & Valor $\mathrm{p}$ & $\mathrm{GdL}$ & Estadístico & $\begin{array}{c}\text { Desviación } \\
\text { Estándar } \\
1 \text { vs } 2\end{array}$ \\
1 & 1 & 0,0181 & 4 & 7,3270 & 0,0346 \\
1 vs 3 & 1 & 0,0000988 & 4 & 1,5919 & 0,0303 \\
2 vs 3 & 1 & 0,0089 & 4 & 4,7624 & 0,046 \\
4 vs 5 & 1 & 0,0002239 & 4 & 12,6636 & 0,0255 \\
4 vs 6 & 1 & 0,00003769 & 4 & 19,8915 & 0,0241 \\
5 vs 6 & 1 & 0,01 & 4 & 4,6026 & 0,0340 \\
7 vs 8 & 1 & 0,0012 & 4 & 8,1505 & 0,0536 \\
7 vs 9 & 1 & $0,0318 \cdot 10-7$ & 4 & 66,6732 & 0,0075 \\
8 vs 9 & 0 & 0,3024 & 4 & 1,1828 & 0,0531 \\
\hline \hline
\end{tabular}

Como se puede observar en las dos tablas anteriores, existe una mayor influencia de la longitud de fibra, demostrándose que existen diferencias en la humedad final, ya que se rechazan todas las pruebas de hipótesis excepto para la comparación 8 y 9 en los cuales no es posible rechazar la hipótesis de igualdad entre las medias. Sin embargo, esta igualdad en las medias de las humedades finales se puede deber a que para el tratamiento 9 , si bien hay una mayor gradiente en la caída de la temperatura, 
se observa que alcanza la humedad final en un tiempo inferior a los 180 min establecidos para el experimento.

\section{A. Perfiles de humedad}

La cinética de secado constituye un factor importante cuando se requiere evaluar la eficiencia del proceso de secado. De manera que se desarrolló una comparación de la cinética de secado para cada uno de los tratamientos experimentales (consistentes en una combinación de longitud de fibra y temperatura del medio de secado). La influencia de la longitud de fibra se puede observar en las Fig. 4 a 6, mientras que la influencia de la temperatura del medio de secado se puede apreciar en las Fig. 7 a 9.

\section{1) Influencia de la longitud de la fibra}

A partir de las curvas se puede ratificar el hecho que la longitud de fibra tiene mayor influencia no solo en la humedad final, sino también en la cinética de secado. Como se puede observar para el nivel inferior de la longitud de fibra $(\mathrm{L}=6 \mathrm{~mm})$, se observa una tendencia leve a la reducción de la humedad para cada una de las temperaturas evaluadas, así como una humedad final elevada. Dicho comportamiento va cambiando a medida que la longitud de fibra aumenta, presentándose comportamientos cada vez más cercanos tanto en la cinética de secado como en la humedad final.

La influencia significativa del tamaño de fibra se puede deber principalmente a que, a mayor longitud de fibra, y por ende mayor sección transversal, existe un mejor contacto del medio de secado con la superficie del material a secar. El flujo de aire puede circular mejor entre los espacios libres al interior de la biomasa. De esta forma el área de contacto efectiva aumenta y por ende la tasa de transferencia de la humedad.

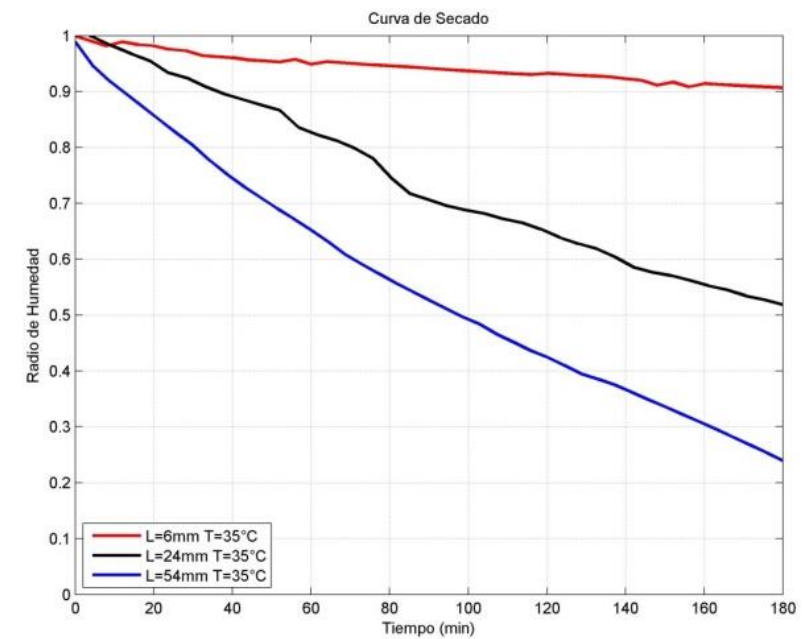

Fig. 4. Perfil de humedad para temperatura constante de $35^{\circ} \mathrm{C}$ del flujo de secado.

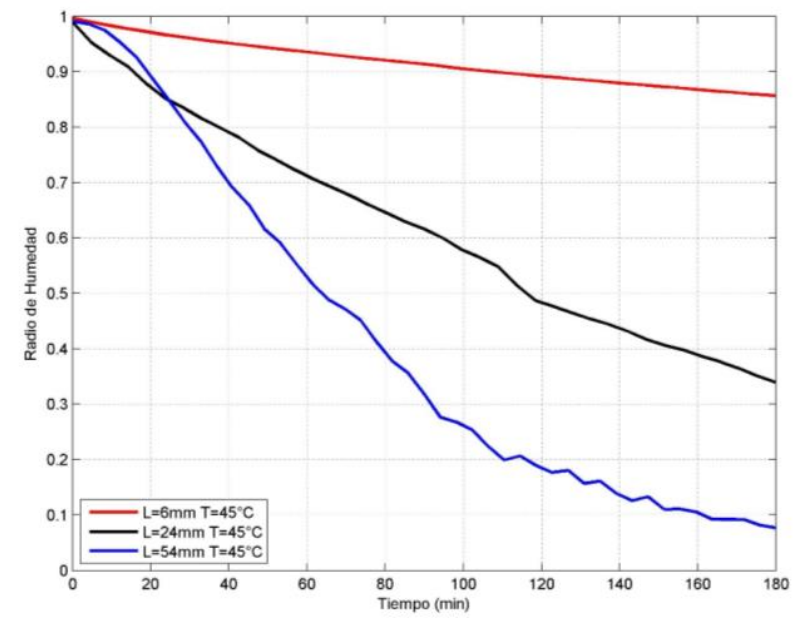

Fig. 5. Perfil de humedad para temperatura constante de $45^{\circ} \mathrm{C}$ del flujo de secado.

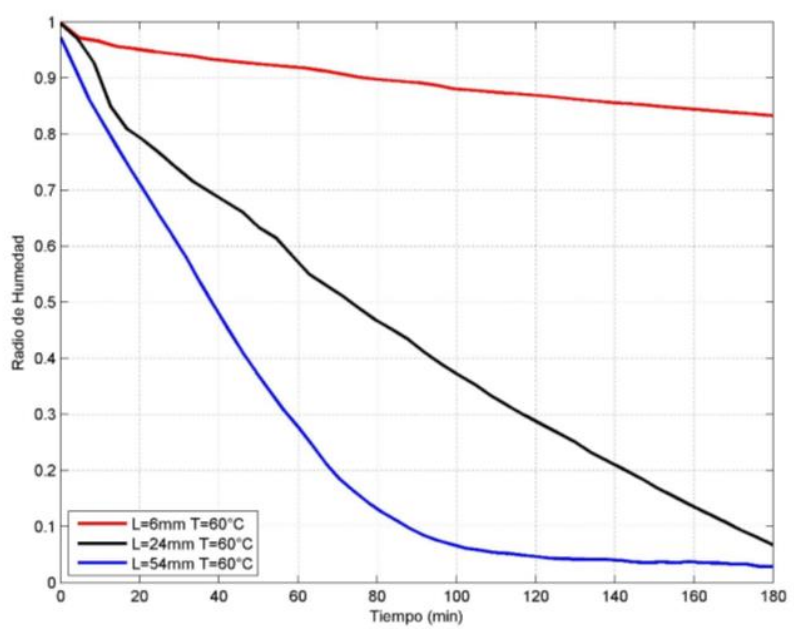

Fig. 6. Perfil de humedad para temperatura constante de $60^{\circ} \mathrm{C}$ del flujo de secado.

\section{2) Influencia de la temperatura del medio de secado}

Por otra parte, para una longitud de fibra constante, se observa una menor influencia de la temperatura en la cinética de secado, al presentarse menores variaciones tanto en la humedad final como en la cinética de secado.

Se observa que la única prueba que alcanzó la humedad de equilibrio fue la combinación $\mathrm{T}=60^{\circ} \mathrm{C}$ y L $=54 \mathrm{~mm}$, en donde se logró una humedad muy cercana a la humedad final en un tiempo $\mathrm{t}=120 \mathrm{~min}$, lo cual corresponde a tres cuartas partes del tiempo total del experimento. Este dato es importante dado que nos permite determinar la viabilidad de realizar el secado de varios lotes el mismo día. Con lo cual se podría lograr una disminución significativa del tamaño de la unidad de secado en campo. 


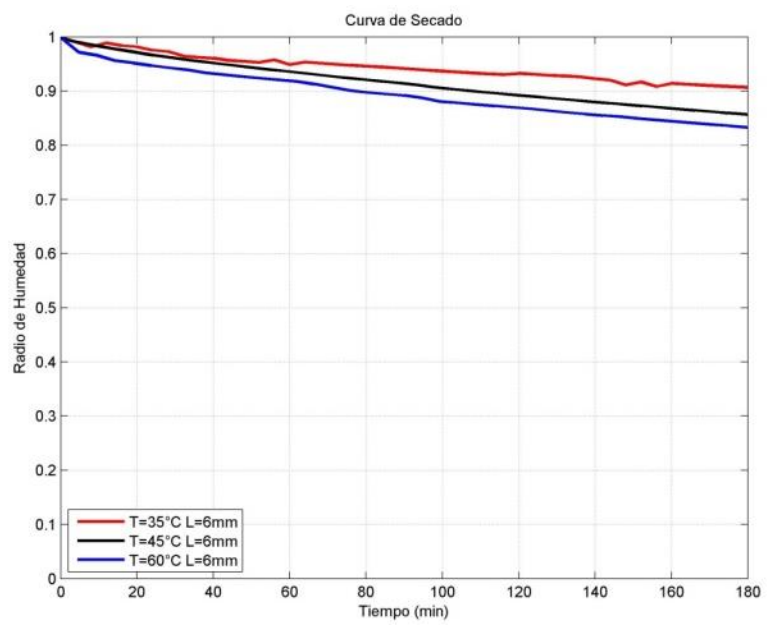

Fig. 7. Perfil de humedad para una longitud de fibra constante de $6 \mathrm{~mm}$.

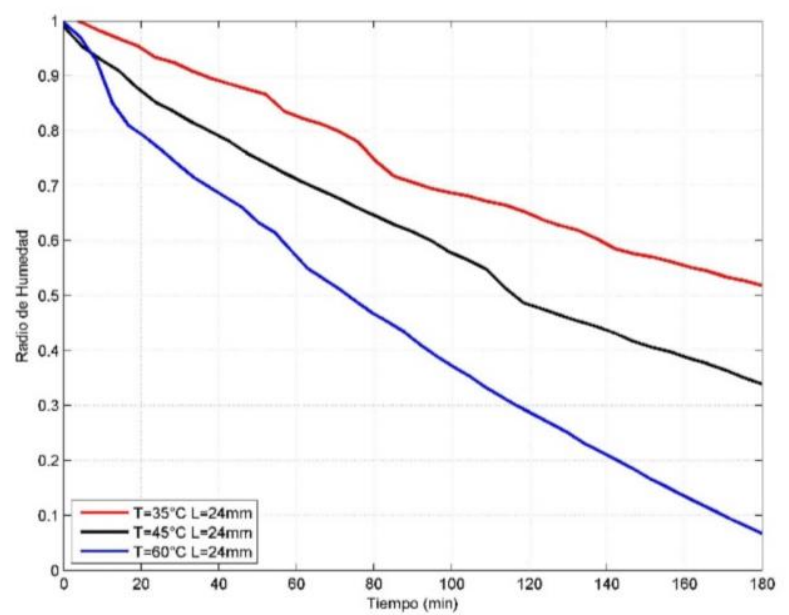

Fig. 1. Perfil de humedad para una longitud de fibra constante de $24 \mathrm{~mm}$.

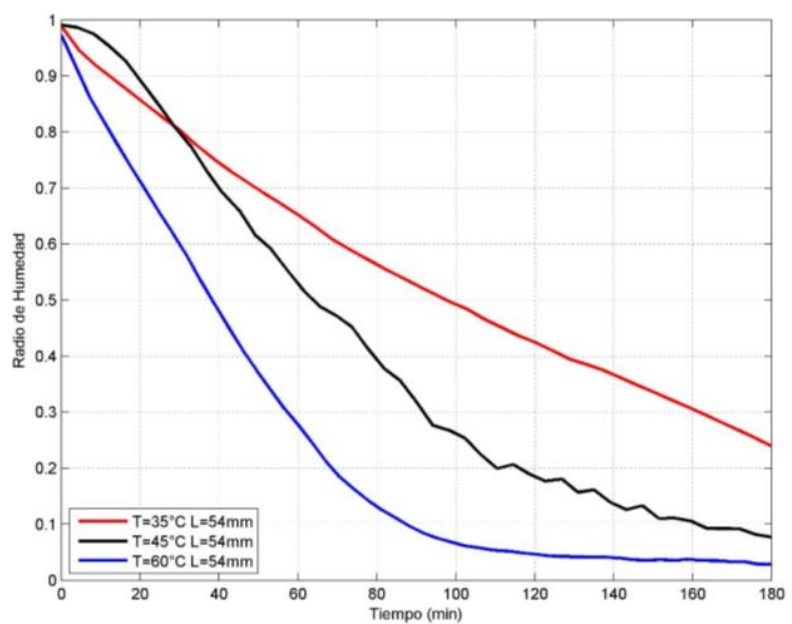

Fig. 9. Perfil de humedad para una longitud de fibra constante de $54 \mathrm{~mm}$.

\section{B. Análisis del experimento}

Los resultados experimentales fueron analizados mediante un software estadístico especializado, determinando de esta forma un modelo matemático experimental que permite determinar la humedad final para diversos valores de los parámetros del proceso de secado. Adicional a esto se determina cuál de las variables estudiadas tiene mayor influencia en el resultado final de la humedad. El análisis se hizo mediante la obtención de una superficie de respuesta arrojando los resultados que a continuación se presentan.

En la Fig. 10 se muestra el diagrama de efectos principales para el diseño experimental ejecutado, donde se demuestra que existe una mayor influencia de la longitud de fibra. Adicional a esto en la Fig. 11 se aprecia que existe además una influencia reducida entre la interacción de los factores experimentales. Adicional a esto se evidencia que a medida que aumenta el tamaño de la fibra, disminuye la humedad final de la fibra. Sin embargo, este comportamiento tiende a variar para una longitud de fibra superior a $50 \mathrm{~mm}$.

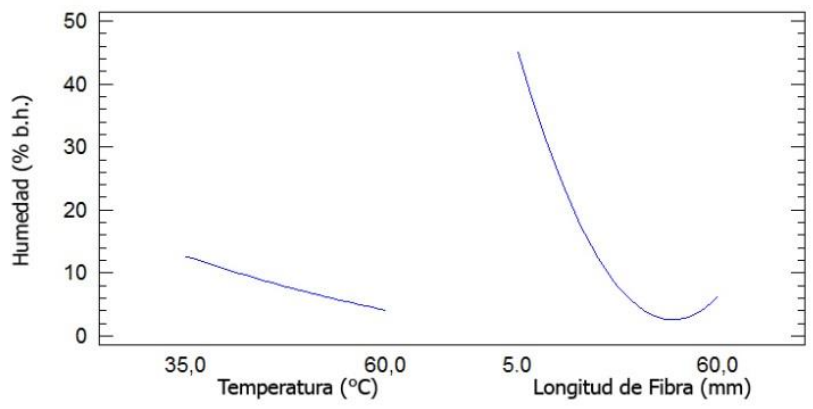

Fig. 10. Diagrama de efectos principales para el contenido de humedad.

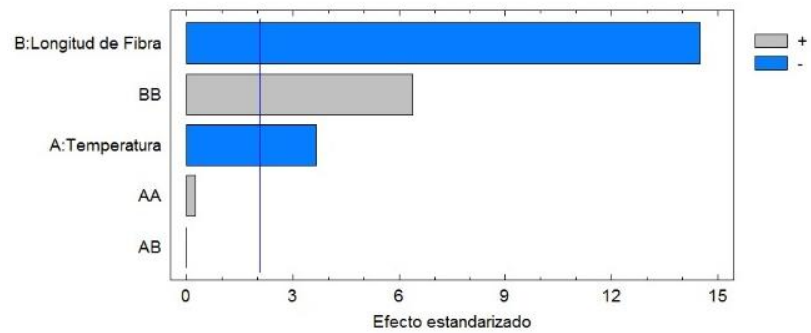

Fig. 11. Diagrama de Pareto estandarizado para el contenido de humedad.

Por último, en la Fig. 12 se muestra la superficie de respuesta para el contenido de humedad (W), la cual se expresa de acuerdo con la siguiente ecuación,

$W=72,296-0,649 T-2,243 L+0,0032 T^{2}+$
$+0,0237 \mathrm{~L}^{2}+1,909 \cdot 10^{-6} T L$

Este modelo ajustado permite explicar el $94,54 \%$ de la variabilidad presentada en la humedad final de la biomasa.

Los resultados obtenidos muestran que es posible mejorar la eficiencia de secado a partir de una combinación adecuada de longitud de fibra y temperatura del medio de secado. De esta forma es posible aumentar la velocidad de secado, o disminuir la energía específica consumida dependiendo de la combinación de los parámetros del proceso de secado. 


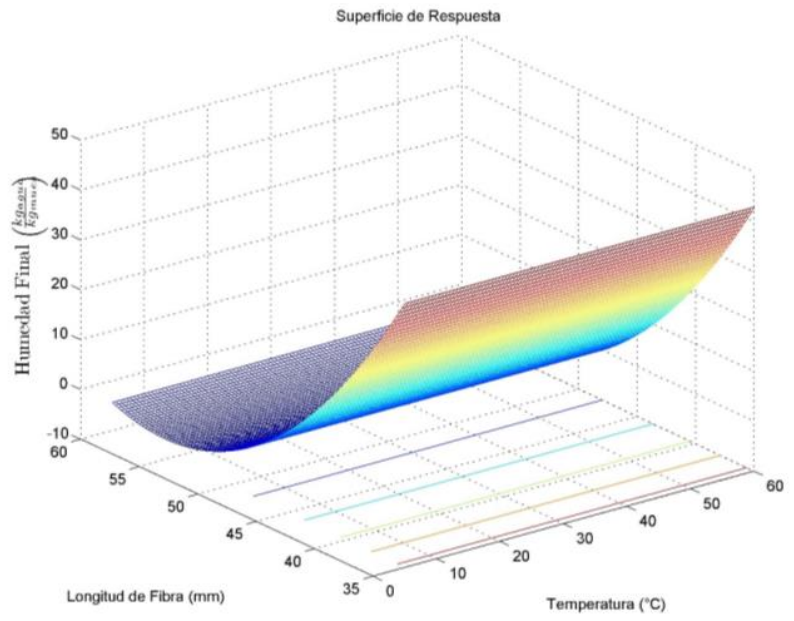

Fig. 12. Superficie de respuesta para el contenido de humedad final.

Se señala además que el rango de temperaturas utilizado en la presente investigación corresponde al que podría ser alcanzado con energía solar como principal fuente de energía. Por ello, los resultados obtenidos son compatibles con el desarrollo de una tecnología de secado agroindustrial basada en colectores solares como medio de calentamiento del aire de secado.

El estudio de la cinética de secado de bagazo de caña en pila o colchón constituye un avance significativo a la hora de mejorar la productividad de la producción de panela en pequeños y medianos trapiches en Colombia y en otras partes del mundo. Estos resultados cobran importancia debido a la falta de estudios experimentales del secado en pila o colchón del bagazo de caña de azúcar panelera. Dado que antes de desarrollar secadores en campo, es necesario tener información suficiente sobre la cinética de secado en pila de esta biomasa.

\section{Comparación de resultados}

Los perfiles de humedad obtenidos experimentalmente se comparan con algunos de los modelos matemáticos usados en la determinación de cinética de secado de material biológico. Para ello se usaron cuatro modelos, el modelo de Chauhan que es usada para el estudio de secado en lecho profundo; y los modelos de Luikov, Newton y Henderson Pabis, los cuales son usados para el estudio de secado en lecho fijo. Estos modelos fueron resueltos mediante el método de las diferencias finitas y se empleó para ello un dominio de secado bidimensional de acuerdo con el esquema mostrado en la Fig. 13.

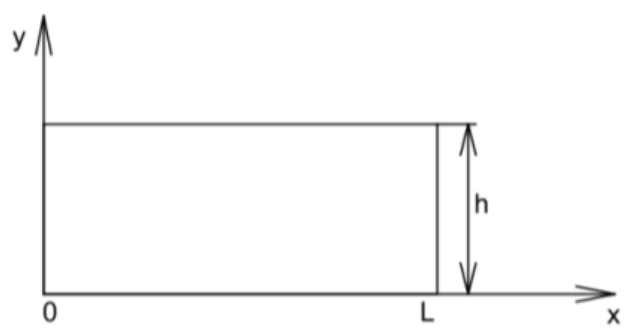

Fig. 13. Esquema bidimensional adoptado para modelamiento.

\section{1) Modelo de Chauhan}

El modelo de Chauhan (2 a 5) es usado para el estudio del secado convectivo en lecho fijo. En la Fig. 14 se puede observar el contraste de las curvas experimentales y las teóricas.

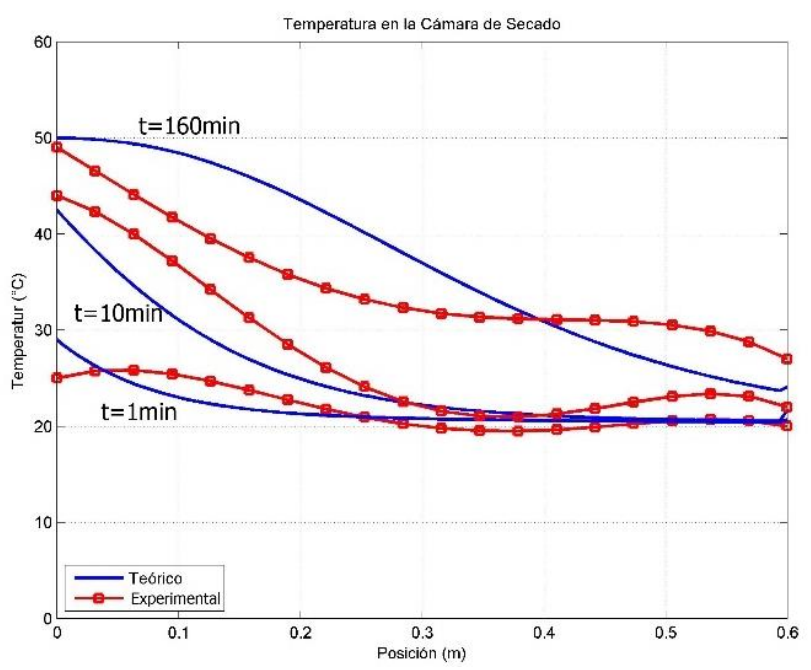

Fig. 14. Comparación entre los perfiles de temperatura experimentales y mediante el modelo de Chauhan.

$$
\begin{gathered}
\rho_{f} \varepsilon_{G B}\left(C_{f}+C_{v} \lambda_{a}\right) \frac{\partial T_{f}}{\partial t}+G_{G B}\left(C_{f}+C_{v} \lambda_{a}\right) \frac{\partial T_{f}}{\partial x} \\
=h_{v G R}\left(T_{G R}-T_{f}\right) \\
\rho_{G R}\left(C_{G R}+C_{w} M_{k t}\right) \frac{d T_{G R}}{\partial t}+h_{f g} G_{G R} \frac{d \lambda_{a}}{d x}= \\
h_{v f G R}\left(T_{f}-T_{G R}\right) \\
\frac{d \lambda_{a}}{d x}=-\frac{\rho_{G R}}{G_{G B}} \frac{d M_{k t}}{d T} \\
\frac{d M_{t}}{d t}=-K_{d}\left(M_{k t}-M_{k e}\right)
\end{gathered}
$$

\section{Modelo de Luikov modificado}

El modelo de Luikov modificado (6 a 8) es uno de los principales modelos fenomenológicos presentes en la bibliografía especializada, usada para el estudio de la cinética de secado en capa delgada. El contraste de los resultados se presenta en la Fig. 15.

$$
\begin{gathered}
C_{q} \frac{\partial T}{\partial t}=K_{11} \nabla^{2} T+K_{12} \nabla^{2} M_{a}+K_{13} \nabla^{2} P \\
C_{m} \frac{\partial M_{a}}{\partial t}=K_{21} \nabla^{2} T+K_{22} \nabla^{2} M_{a}+K_{23} \nabla^{2} P \\
C_{p} \frac{\partial P}{\partial t}=K_{31} \nabla^{2} T+K_{32} \nabla^{2} M_{a}+K_{33} \nabla^{2} P
\end{gathered}
$$




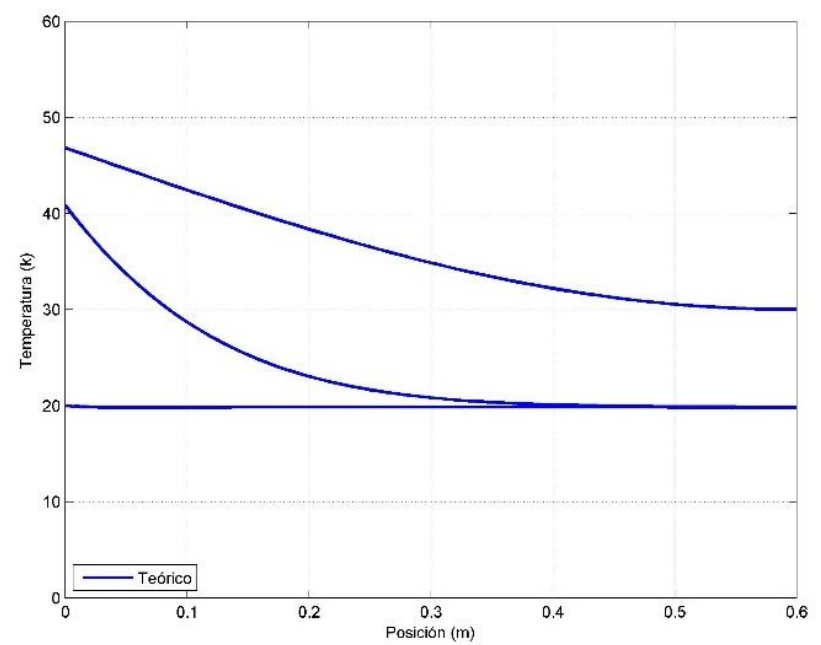

(a)

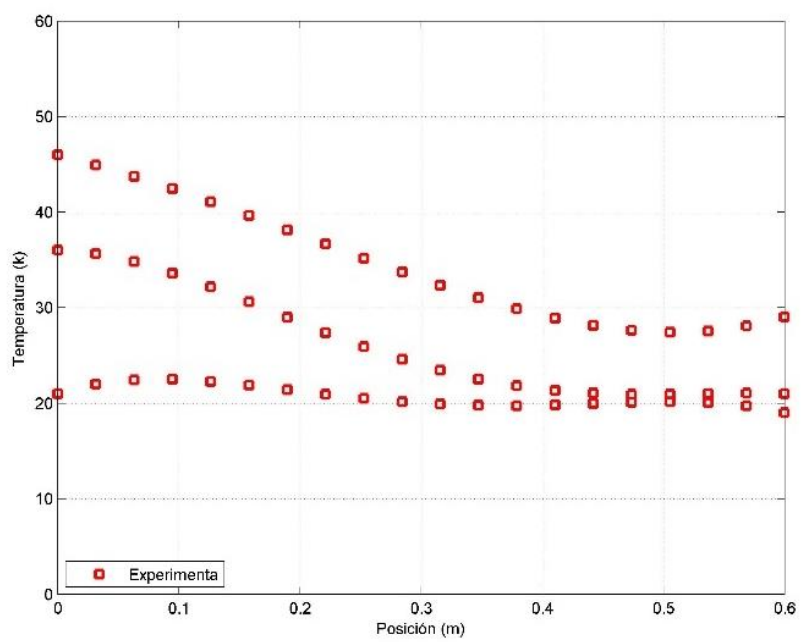

(b)

Fig. 15. Contraste entre los resultados teóricos del modelo de Luikov modificado (a) y los datos experimentales (b).

\section{2) Modelo de Newton}

El modelo de Newton (9) es el más simple para capa delgada, dado que involucra una variable adimensional. En la Tabla 8 se presentas los valores de la constante de correlación k. En la Fig. 16 se presenta el contraste entre los resultados teóricos y experimentales, para el tratamiento experimental de $35^{\circ} \mathrm{C}$ y longitud de fibra de $6 \mathrm{~mm}$.

$$
M R=e^{-k t}
$$

TABLA VIII

APROXIMACIÓN DE RESULTADOS EXPERIMENTALES AL MODELO DE NEWTON

\begin{tabular}{ll}
\hline \hline Combinación & $\mathrm{k}$ \\
$\mathrm{T}=35^{\circ} \mathrm{C}$ y $\mathrm{L}=6 \mathrm{~mm}$ & 0,00060 \\
$\mathrm{~T}=35^{\circ} \mathrm{C}$ y $=24 \mathrm{~mm}$ & 0,00085 \\
$\mathrm{~T}=35^{\circ} \mathrm{C}$ y $\mathrm{L}=54 \mathrm{~mm}$ & 0,00090 \\
\hline \hline
\end{tabular}

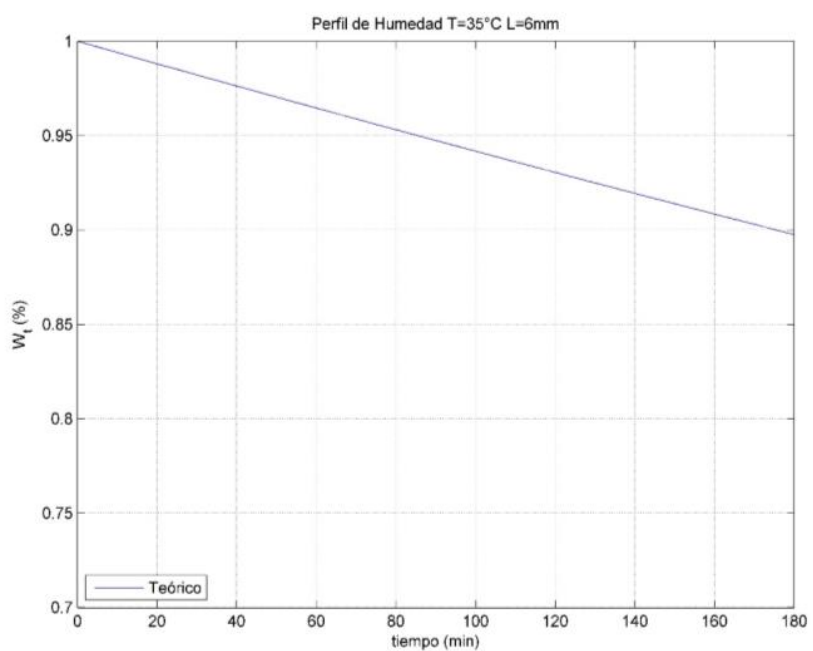

(a)

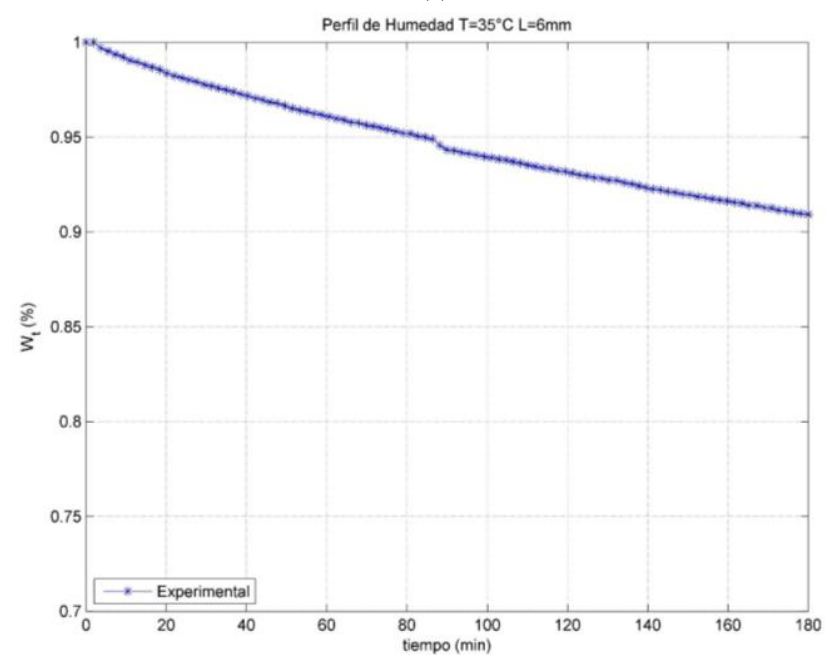

(b)

Fig. 16. Contraste entre los resultados teóricos del modelo de Newton (a) y los datos experimentales (b), para el tratamiento experimental de $35^{\circ} \mathrm{C}$ y longitud de fibra de $6 \mathrm{~mm}$.

\section{3) Modelo de Henderson Pabis}

El modelo de dos términos (10) es usualmente empleado para el modelamiento del fenómeno de secado. Su formulación es sencilla y depende de dos factores adimensionales. En la Tabla 9 se muestran los valores de las constantes $\mathrm{k}$ usadas para la solución del modelo. En la Fig. 17 se muestra el contraste de los resultados para el tratamiento experimental de $35^{\circ} \mathrm{C}$ y longitud de fibra de $54 \mathrm{~mm}$.

$$
M R=a e^{-k_{0} t}+b e^{-k_{1} t}
$$

TABLA IX

CONSTANTES ADIMENSIONALES PARA EL MODELO DE HENDERSON PABIS

\begin{tabular}{lll}
\hline Combinación & $\mathrm{k}_{0}$ & $\mathrm{k}_{1}$ \\
$\mathrm{~T}=35^{\circ} \mathrm{C}$ y $\mathrm{L}=6 \mathrm{~mm}$ & 0,0008 & 0,0008 \\
$\mathrm{~T}=35^{\circ} \mathrm{C}$ y $\mathrm{L}=24 \mathrm{~mm}$ & 0,0032 & 0,0032 \\
$\mathrm{~T}=35^{\circ} \mathrm{C}$ y $\mathrm{L}=54 \mathrm{~mm}$ & 0,0072 & 0,0073 \\
\hline
\end{tabular}




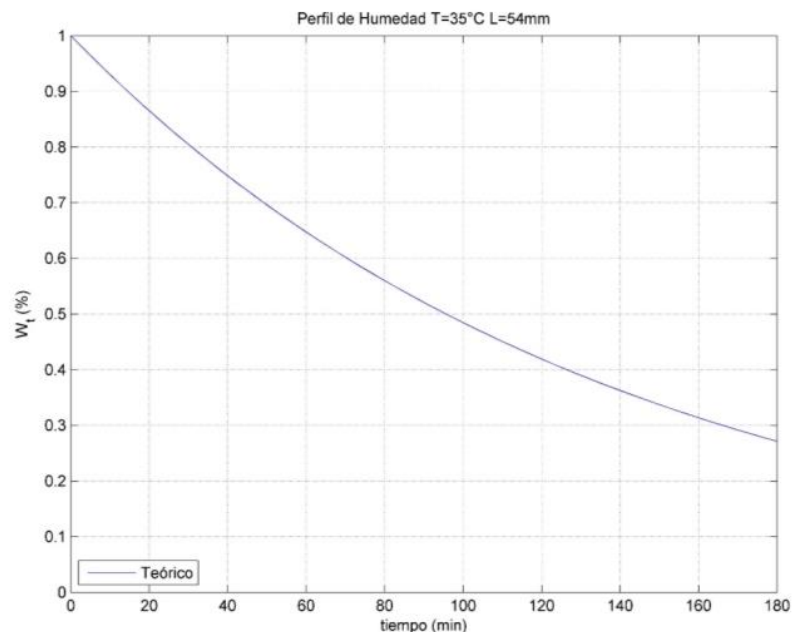

(a)

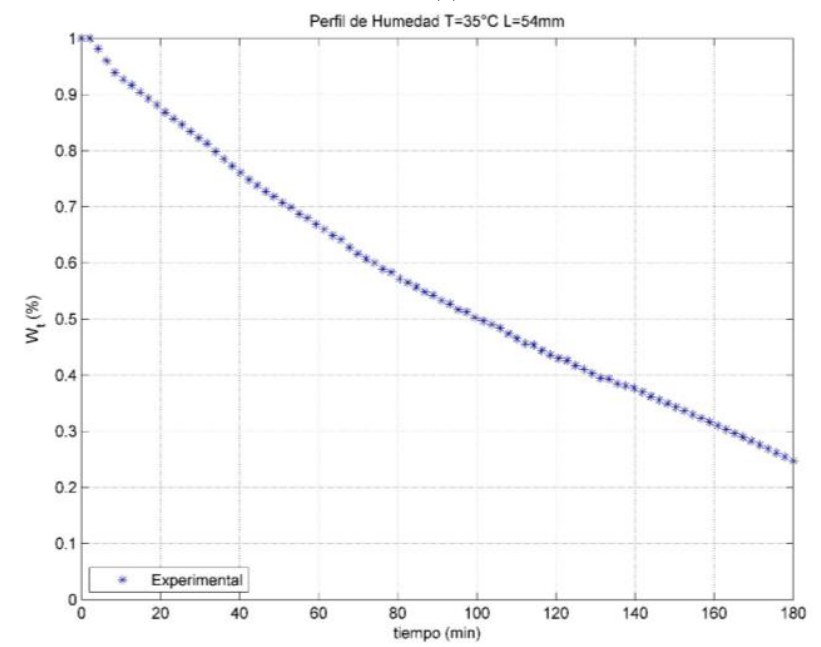

(b)

Fig. 17. Contraste entre los resultados teóricos del modelo de Henderson Pabis (a) y los datos experimentales (b), para el tratamiento experimental de $35^{\circ} \mathrm{C}$ y longitud de fibra de $54 \mathrm{~mm}$.

\section{CONCLUSIONES}

El estudio de cinética de secado de diversos materiales y mediante el uso de diferentes tecnologías de secado, en especial aquellos vitales para los sistemas productivos regionales, se constituye en un punto de partida para el desarrollo de modelos unificados de predicción de la cinética de secado.

Los modelos estadísticos experimentales obtenidos en el presente trabajo presentan una buena relación con los resultados reportados por otros autores. Por otra parte, los resultados de humedad final también se encuentran cerca a los presentados en la bibliografía. Sin embargo, industrialmente los procesos normalmente no alcanzan humedades tan bajas. De esta forma Sosa-Arnao et al. [10] presentan una revisión de investigaciones y aplicaciones de secado de bagazo de caña las cuales alcanzan humedades finales en promedio superiores a $35 \%$ en b.h. Sin embargo, es necesario anotar que estos modelos presentan resultados óptimos bajo un rango de condiciones estrecho; lo anterior debido a que para pequeñas variaciones de los parámetros se presenta variaciones significativas en los datos recopilados.
Desde el comienzo de este estudio se contempló el desarrollo de una unidad experimental novedosa para tal fin, dado que no se contaba con un equipo que se adecuara a las condiciones requeridas para el desarrollo de la presente investigación. De esta forma se desarrolló el proceso de diseño y construcción de un secador experimental, el cual cuenta con una precisión adecuada para este tipo de pruebas, contando con una resolución estrecha: $\pm 1 \mathrm{~g}$ para peso, $\pm 1^{\circ} \mathrm{C}$ para temperatura $\mathrm{y}$ $\pm 1 \%$ HR. Adicional a esto cuenta con una frecuencia de muestreo excelente para el fenómeno en estudio (ajustable entre $0,1-1 \mathrm{~Hz}$ ); lo cual es adecuado para el estudio de la cinética de secado convectivo en lecho fijo, dado que involucra tiempos extensos.

A la luz de los resultados alcanzados se puede afirmar que es posible obtener resultados óptimos de secado del bagazo de caña panelera usando equipos de baja temperatura y relativa baja potencia. Lo cual haría posible la implementación de este tipo de secadores con un costo de instalación y mantenimiento asequibles al sector agroindustrial panelero colombiano. Como se observa en los resultados obtenidos, con temperaturas que oscilan entre $35^{\circ} \mathrm{C}$ y $60^{\circ} \mathrm{C}$ y longitudes de fibra entre $24 \mathrm{~mm}$ y $54 \mathrm{~mm}$ respectivamente, se pueden alcanzar humedades finales adecuadas, tanto para el proceso de combustión como para la densificación de esta biomasa con fines de almacenamiento o comercialización. Ahora bien, si el bagazo panelero es requerido con una humedad final que oscile entre $10 \%$ y $20 \%$ en b.h., no se recomienda usar temperaturas por debajo de $45^{\circ} \mathrm{C}$ y longitudes de fibra menor a $24 \mathrm{~mm}$.

Es posible aumentar la eficiencia del proceso de secado, controlando el contacto del medio de secado con el material, el cual de acuerdo con los resultados obtenidos es el principal factor que influye en la cinética de secado. De esta forma, cuando el tamaño de la fibra es menor a $24 \mathrm{~mm}$, el material procesado dificulta el flujo del aire a través del lecho de secado; esto a su vez ocasiona una reducción importante en la eficiencia de secado. Lo anterior se evidencia en las gráficas de humedad final, en las cuales se muestran diferencias significativas en la humedad final entre el tamaño de fibra de $6 \mathrm{~mm}$ y las otras dos longitudes de fibra definidas en el presente estudio.

Otra característica importante que se observa es que el tamaño de fibra tiene una mayor influencia, que la temperatura, sobre la humedad final de la muestra. Dicho comportamiento puede ser atribuido al bajo gradiente de presión generado por un ventilador axial, lo cual ocasiona un menor flujo de aire entre las fibras para longitudes menores. De las curvas obtenidas para la humedad final en función de la temperatura y la longitud de la fibra se evidencia que la influencia de la temperatura del medio de secado sobre la humedad final tiende a ser lineal, mientras que la influencia de la longitud de fibra se adecúa mejor a un comportamiento cuadrático.

Para una temperatura de $60^{\circ} \mathrm{C}$ y una longitud de fibra de $54 \mathrm{~mm}$, se observa una tasa de secado alta, dado que se obtiene la humedad final en un tiempo de $120 \mathrm{~min}$. Lo que corresponde a alrededor de las tres cuartas partes del tiempo estimado para el experimento. Sin embargo, la humedad final alcanzada es similar a la que se presenta para combinaciones de temperatura y longitud de fibra de $45^{\circ} \mathrm{C}$ y $54 \mathrm{~mm}$ y también para $60^{\circ} \mathrm{C}$ y 24 $\mathrm{mm}$. Esto se comprueba en los resultados de la prueba de Student, en donde se comprueba que no hay diferencias 
estadísticamente significativas entre las medias de los resultados.

Para la combinación de temperatura de $60^{\circ} \mathrm{C}$ y un tamaño de fibra de $54 \mathrm{~mm}$, se observa una marcada tendencia a detenerse el proceso de secado cerca al 3\% de humedad (b.h.) (humedad de equilibrio). Este mismo comportamiento se observa para temperatura de $45^{\circ} \mathrm{C}$ y longitud de fibra $54 \mathrm{~mm}$, el cual tiende a estabilizarse alrededor de $8 \%$ de humedad (b.h.).

$\mathrm{Si}$ bien los mejores resultados del proceso de secado se obtuvieron con la combinación $\mathrm{T}=60^{\circ} \mathrm{C}$ y $\mathrm{L}=54 \mathrm{~mm}$, con la cual se consiguió una humedad promedio cercana al $2 \%$ (b.h.), se tiene que para la combinación $\mathrm{T}=35^{\circ} \mathrm{C}$ y $\mathrm{L}=54 \mathrm{~mm}$ se presenta una mayor eficiencia térmica, con un consumo específico de energía de aproximadamente la mitad.

El presente estudio del secado de bagazo de caña en pila constituye un importante aporte para la comprensión de este proceso aplicado al sector rural. Dado que en la actualidad los estudios relacionados se centran fundamentalmente en otros mecanismos de secado, con tecnologías inapropiadas para el sector rural colombiano, debido principalmente al nivel de innovación tecnológica requerido, la complejidad constructiva, su mantenimiento y las características de operación de estas. De esta forma, los resultados obtenidos sobre la cinética del secado de bagazo de caña panelera en pila verifican la factibilidad de alcanzar humedades finales convenientes; y se constituyen en un avance significativo para en un futuro lograr escalar esta tecnología y hacerla llegar al consumidor agroindustrial final.

\section{REFERENCIAS}

[1] J. H. Rodríguez, and P. Quinto Diez, "Secado de medios porosos: una revisión a las teorías actualmente en uso," Científica, vol. 9, no. 2, pp. 63-71, 2005, ISSN1665-0654.

[2] L. Fagernäs, J. Brammer, C. Wilén, M. Lauer, and F. Verhoeff, "Drying of biomass for second generation synfuel production," Biomass and Bioenergy, vol. 34, pp. 1267-1277, 2010, DOI: 10.1016/j.biombioe.2010.04.005.

[3] S. Pang, and A. S. Mujumdar, "Drying of Woody Biomass for Bioenergy: Drying Technologies and Optimization for an Integrated Bioenergy Plant,” Dry. Technol., vol. 28, pp. 690-701, 2010, DOI: 10.1080/07373931003799236.

[4] H. Li, Q. Chen, X. Zhang, K. N. Finney, V. N. Sharifi, and J. Swithenbank, "Evaluation of a biomass drying process using waste heat from process industries: A case study," Appl. Therm. Eng., vol. 35, pp. 71-80, Mar. 2012, DOI: 10.1016/j.applthermaleng.2011.10.009.

[5] E. C. Rada, M. Ragazzi, L. Fiori, and D. Antolini, "Bio-drying of grape marc and other biomass: a comparison," Water Sci. Technol., vol. 60, pp. 1065-1071, 2009.

[6] J. A. Villalba, and N. Arzola, "Modelos matemáticos y experimentales sobre el secado de biomasa," Revista Científica Ingeniería y Desarrollo, vol. 33, no. 2, pp. 301-330, 2015, DOI: 10.14482/inde.33.2.5674.

[7] T. Bartzanas, D. D. Bochtis, C. G. Sørensen, A. A. Sapounas, and O. Green, "A numerical modelling approach for biomass field drying," Biosyst. Eng., vol. 106, no. 4, pp. 458-469, 2010, DOI: 10.1016/j.biosystemseng.2010.05.010.

[8] T. Ivanova, B. Havrland, V. Pobedinsky, and A. Muntean, "Analysis of System of Biomass Drying By Use Of Experimental Dryer," Energy Rural Dev., vol. 26, pp. 336-342, 2011.
[9] M. A. Mazutti, G. Zabot, G. Boni, A. Skovronski, D. de Oliveira, M. Di Lucioano, J. V. Oliveira, M. I. Rodrigues, H. Treichel, and F. Maugeri, "Mathematical modeling of thin-layer drying of fermented and non-fermented sugarcane bagasse," Biomass and Bioenergy, vol. 34, pp. 780-786, 2010, DOI: 10.1016/j.biombioe.2010.01.021.

[10] J. H. Sosa-Arnao, F. M. De Oliveira, J. L. G. Corrêa, M. A. Silva, and S. A. Nebra, "Sugar cane bagasse drying - a review," Drying, vol. B, pp. 990-997, 2004.

[11] K. S. Jairaj, S. P. Singh, and K. Srikant, "A review of solar dryers developed for grape drying," Sol. Energy, vol. 83, no. 9, pp. 16981712, 2009, DOI: 10.1016/j.solener.2009.06.008.

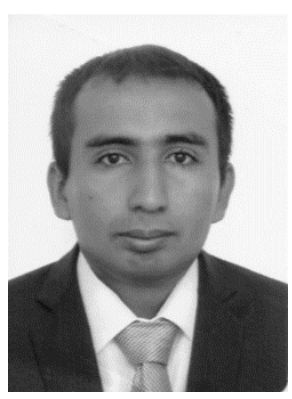

Jorge Armando Villalba Vidales was born in Purificación, Tolima, Colombia in 1984. He received the B.S. degree in Mechanical Engineering from the Universidad Distrital Francisco José de Caldas and M.Sc. from Universidad Nacional de Colombia in 2015. From 2009 to 2014 work in a nonprofit NGO Innovar suroriente in rural development. Since 2017 has been lecturer with the Faculty of Engineering and Basic Science of the Fundación Universitaria Los Libertadores. Hi is the author of one article and a scientific divulgation book.

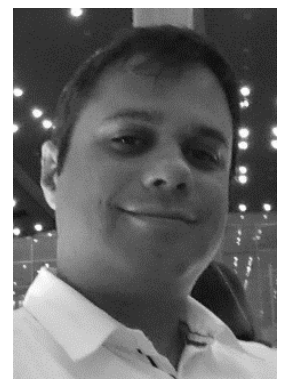

Nelson Arzola de la Peña received the B.S. degree in Mechanical Engineering from Universidad de Cienfuegos, Cienfuegos, Cuba, in 1997; the M.S. degree in Applied Mathematics from Universidad de Cienfuegos, Cienfuegos, Cuba, in 1999; and the Ph.D. degree in Technical Sciences from Universidad Central de las Villas, Santa Clara, Cuba, in 2004.

From 2005 untill now, he is Professor at the Universidad Nacional de Colombia. He is the author of two books, more than 25 scientific papers, and more than 20 conference papers. His research interest includes mechanical design methodology, failure analysis, fracture mechanics, materials and processing and use of biomass. 